\title{
CONTRIBUTION À LA CONNAISSANCE DE LA FLORE VASCULAIRE DE LA FORÊT DE L'ACHACH, PLATEAU CENTRAL (MAROC)
}

\author{
Jamal BAMMI et Allal DOUIRA
}

RÉSUMÉ. L'analyse de la diversité floristique de la forêt de 1'Achach a révélé 1'existence de 269 taxons appartenant à 61 familles et 181 genres. 7 espèces sont endémiques du territoire Maroc-Algérie, six espèces endémiques du territoire Maroc-Péninsule ibérique et deux espèces endémiques du territoire Maroc- Algérie- Tunisie. Trois espèces sont menacées, trois espèces rares et deux espèces soupçonnées rares.

Mots clés: Forêt de l'Achach (Plateau central), Maroc, flore.

RESUMEN. El análisis de la diversidad florística del bosque de l' Achach revela la presencia de 269 taxa pertenecientes a 61 familias y 181 géneros. 7 especies son endémicas de Marruecos-Algeria, seis de Marruecos-Península Ibérica y dos de Marruecos-Algeria-Tunez. Tres especies se encuentran amenazadas, tres son raras y dos dudosamente raras.

Palabras clave. Bosque de l'Achach, Marruecos, flora.

\section{INTRODUCTION}

En comparaison avec les autres pays du Maghreb, le Maroc reste le plus riche de point de vue richesse floristique. La flore algérienne est représentée actuellement par 3000 espèces et 1000 genres (Hanifi, 1991), celle de la Tunisie compte 2103 espèces et 742 genres (Nabli , 1991), alors que la flore totale marocaine est représentée actuellement par 4200 espèces et sousespèces avec 940 genres et 135 Familles (Ibn Tatou et Fennane, 1991).

L'étude que nous allons présenter s'intéresse à un recensement des espèces végétales de la forêt de l'Achach. Elle vient pour enrichir nos connaissances sur les formations végétales de la classe des Quercetea ilicis et surtout pour celles qui se développent en milieu fragile et subissant une forte pression humaine.

\section{MATERIEL ET METHODES}

La région de l'Achach est rattachée dans sa quasi-totalité à la province de Settat. Elle est limitée au Nord et à l'Est, respectivement par les forêts de Benslimane et la forêt de Zaër. Au Sud, elle est limitée par la forêt de Gnadiz, située dans la province de Khouribga.

La région de l'Achach fait partie également du Domaine mesétien et plus 
précisément de la méseta centrale (Michard, 1976). Elle appartient, de point de vue genèse géologique, à l'époque dévonienne et dinantienne (Fadli, 1990).

D'une manière générale, la moitié ouest de la forêt de l'Achach est dominée par les schistes avec intercalations de plaquettes calcaires, des quartzites et grés ainsi que des grès calcareux. Dans la partie Est dominent les flysh gréseux du Khatouat, les flysh schisteux et les schistes calcareux du dévonien moyen et supérieur (Fadli, 1990).

Les sols sur schistes ou quartzites de la région de l'Achach se caractérisent par une pierrosité croissante avec une profondeur variable selon l'exposition ou la pente.

L'analyse du terrain a permis de décerner trois types de sols (Charafi, 1985): des sols peu évolués érodés peu profonds, des sols plus ou moins brunifiés en surface rougeâtres plus bas et des sols minéraux bruts très superficiels et squelettiques.

Dans la région de l'Achach, et plus précisément dans la station de Khatouat, la température moyenne annuelle varie entre $17^{\circ} \mathrm{C}$ et $18^{\circ} \mathrm{C}$. la moyenne des températures maximales du mois le plus chaud atteint la valeur de $32,3^{\circ} \mathrm{C}$ (Combe, 1975). La moyennes des températures minimales du mois le plus froid atteint la valeur de $5,2^{\circ} \mathrm{C}$. La pluviométrie moyenne annuelle est de l'ordre de $543 \mathrm{~mm}$. Le Climagramme d'Emberger permet de situer notre zone d'étude dans l'ambiance bioclimatique semiaride à hiver doux.

De point de vue de la végétation, la forêt de l'Achach a pour particularité essentielle, la réunion sur le même espace de trois espèces forestières majeures du Maroc à savoir: le chêne liège (Quercus suber), le chêne vert (Quercus rotundifolia) et le thuya (Tetraclinis articulata). Cette particularité donne à la forêt un intérêt biologique certain pour la conservation in-situ des plantes rares et menacées.
La réalisation de ce catalogue à été faite à partir de travaux de terrain au cours des années 1997, 1998 et 1999 qui nous ont permis de constituer un herbier avec tous les renseignements nécessaires. Des donnés bibliographiques ont été également exploités en plus de l'herbier du département d'Ecologie Végétale de l'Institut Agronomique et Vétérinaire Hassan II de Rabat. Les échantillons témoins sont déposés dans l'herbier de l'Institut Agronomique et Vétérinaire Hassan II de Rabat.

Les informations données dans ce catalogue se présentent dans l'ordre suivant:

Ecologie: Dans cette partie ont été signalées les nécessités écologiques de chaque espèce, le mode de vie et les habitats où elle a été généralement rencontrée.

Etages bioclimatiques: La notion d'étages bioclimatiques s'avère d'un intérêt incontestable pour compléter la répartition des taxons. Les différentes ambiances bioclimatiques retenues sont celles définies initialement par Emberger, il s'agit: $\mathbf{S}$ : Saharien; A: Aride; SA: Semi-aride; SH: Sub-humide; H: Humide; HM: Haute montagne.

L'altitude maximale: Pour chaque espèce, on mentionne l'altitude maximale (disponible) atteinte par l'espèce dans l'ensemble du Maroc.

Répartition dans la région d'étude: là on mentionne les différentes localités géographiques de la forêt de l'Achach où se trouve l'espèce en question. Les localités en question sont les suivantes:AD: Addalâ; AG: Ain dar ghbar; AS: Assfi ; BA: Batteh; BH: Bir hamza; CL: Chaâbat lebtoum; HB: Hajra lbeida; JH: Jbel el hallouf ; KA: Kraker achra; KH: Khatouat; KR: Jbel karri; LM: Lam'asser; MB: Moul bsseila; MG: Mga'ora; NW: Nwiga; OB: Ouled bou m'aiza; OH: Ouled haj hmida; OM: Ouled moumen; OT: Oued atteuch; SB: Sabbab; 
SM: Sidi mimoun; SS: Sidi sbaâ; TA: Tafrant.

Type biologique: $\mathrm{Ph}$ (Phanérophytes), Np (Nanophanérophytes), C (Chaméphytes), H (Hémicryptophytes), G (Géophytes), Th (Thérophytes).

Endémisme: EMA (Endémiques du Maroc et d'Algérie) ; EMI ( Endémiques du Maroc et de la péninsule Ibérique); EMAT (Endémiques du Maroc, Algérie et Tunisie).

Les espèces rares ou menacées: $R R$ : très rare (menacée); R: rare; R ?: soupçonnée rare.

\section{RÉSULTATS}

\section{Anacardiaceae}

\section{Pistacia atlantica Desf.}

Rochers, ravins, Dayas, des steppes, forêts des basses et moyennes montagnes. A, SA, SH. Jusqu'au 2000 m. localités CL, OB, HB, SB, KH, AS, OM, LM, AG. Ph (type biologique).

\section{Pistacia lentiscus L.}

Forêt et maquis de la plaine et des basses montagnes. SA, SH, H. jusqu'au $1600 \mathrm{~m}$. localités SS, MB, OM, JH, OT, SM, MG, OM, OB, CL, HB, KH. Np(Ph)

\section{Rhus pentaphylla (Jacq.)Desf.}

Forêts claires, broussailles de la plaine et des basses montagnes. S, A, SA. Jusqu'au 1000 m. localités SS, SM, TA, OB, CL, HB, NW, AD, SB, KR, KH. Np(Ph)

\section{Apiaceae}

\section{Ammi majus L.}

Broussailles, pâturages, champs de la plaine et des basses montagnes. A, SA, SH, H. localités OA, AG, AS, KH, OH, KA. Th

Bunium alpinum Waldst. \&Kit.

Forêts et pâturages rocailleux des montagnes. SA, SH, H. jusqu'au $2400 \mathrm{~m}$. localité SS. G

\section{Daucus muricatus (L.) L.}

Forêts claires, pâturages, de la plaine et des basses et moyennes montagnes. A, SA, SH, H. localités KH, KA. Th

\section{Elaeoselinum asclepium (L.) Bertol.}

Forêts claires, pâturages sablonneux et rocailleux de la plaine et des montagnes. $\mathrm{SA}, \mathrm{SH}$, H. jusqu'au $2200 \mathrm{~m}$. localités TA, CL, HB, NW, OB, BA, KH, AS, LM, SB, OT, KA, OH. Hém

\section{Elaeoselinum foetidum (L.) Boiss.}

Coteaux secs, terrains sablonneux, broussailles. SA, SH, H. localité non disponible. Hém. EMI.

\section{Eryngium tricuspidatum $\mathrm{L}$.}

Broussailles, coteaux secs, lieux arides. A, $\mathrm{SA}, \mathrm{SH}, \mathrm{H}$. toutes les localités. Hém

\section{Eryngium triquetrum Vahl.}

Pâturages rocailleux, clairières des forêts, champs cultivés de la plaine et des montagnes. A, SA, SH. Jusqu'au 2300 m. localités TA, SS. Hém

\section{Ferula communis L.}

Clairières des forêts, rochers, pâturages sablonneux et rocailleux de la plaine et des montagnes. A, SA, SH, H. jusqu'au $2200 \mathrm{~m}$. localités AD, NW. G

Ferrula lutea (Poiret) M.

Forêts claires, broussailles, pâturages des basses montagnes. SA, SH, H. localités MG, TA, AD, KH. G

\section{Foeniculum vulgare Miller}

Champs incultes, coteaux arides, ravins, rochers de la plaine et des basses montagnes. A, SA, SH, H. jusqu'au $1800 \mathrm{~m}$. localité non disponible. Ch (Hém)

\section{Thapsia garganica L.}

Clairières des forêts, pâturages sablonneux et rocailleux, steppes, dans la plaine et les basses montagnes. A, SA, SH, H. localités SS, JH, MG, OT, TA, CL, KH, LM, OH. G

Torilis arvensis (Hudson) Link 
Forêts claires, Broussailles fraîches, bords des eaux, dans la plaine et les basses et moyennes montagnes. A, SA, SH, H. jusqu'au $1500 \mathrm{~m}$. localité KH. Th

Torilis leptopylla (L.) Reinchenb. Fil.

Moissons, champs, pâturages rocailleux des montagnes. A, SA, SH, H. jusqu'au $2200 \mathrm{~m}$. localité non disponible. Th

\section{Torilis nodosa (L.Graetner.)}

Forêts claires, pâturages pierreux, champs cultivés de la plaine et des basses montagnes. A, SA, SH, H. toutes les localités. Th

\section{Apocynaceae}

Nerium oleander L.

Bords des oueds. S, A, SA, SH, H. jusqu'au 2000 m. localités OT et SB. Nph

\section{Araceae}

\section{Arisarum vulgare Targ-Tozz.}

Lieux ombragés, talus, vieux murs, pâturages rocailleux de la plaines et des basses montagnes. A, SA, SH, H. Toutes les localités. G

\section{Arecaceae}

\section{Chamaerops humilis L.}

Forêts, broussailles, pâturages argileux, sablonneux et pierreux secs de la plaine et des montagnes. A, SA, SH. Jusqu'au $2300 \mathrm{~m}$. localités SM, CL, NW, AD, OB, SB, KH, KR, OM, LM, KA, OT. Ch (Ph)

\section{Aristolochiaceae}

\section{Aristolochia paucinervis Pomel.}

Champs, pâturages pierreux, argileux et sablonneux, forêts. SA, SH, H. jusqu' au 1700m. localités KH, KA. G. EMAT.

\section{Asparagaceae}

\section{Asparagus acutifolius L.}

Forêts et broussailles des plaines et des montagnes. A, SA, SH. Jusqu'au 1900 m. localité SM. G

\section{Asparagus albus L.}

Steppes, broussailles, forêts claires, rochers de la plaine et des basses montagnes. A, SA, SH. Jusqu' au 1500 m. localités TA, CL, SL, KH. Nph (G)

\section{Asparagus altissimus L.}

Broussailles, maquis, roches de la plaine et des basses montagnes. S, A, SA. Localités OB, NW, LM, KH, KA. Nph (G). EMA

\section{Asparagus officinalis L.}

Prairies humides, cultivé et quelquefois subspontané. SH. Localité KH. G

\section{Asparagus stipularis Forsk.}

Steppes, lieux pierreux ou sablonneux arides de la plaine et des montagnes. S, A, SA, SH. Jusqu'au $2000 \mathrm{~m}$. localité non disponible. G

\section{Aspholidaceae}

\section{Asphodelus ramosus L.}

Pâturages rocailleux, maquis des basses et moyennes montagnes. SA, SH, H. jusqu'au 2000 m. toutes les localités. G. EMI.

\section{Asteraceae}

\section{Andryala integrifolia $\mathrm{L}$.}

Clairières des forêts, pâturages, rochers, vieux murs, falaises maritimes, dunes. A, SA, SH, H. jusqu' au $1700 \mathrm{~m}$. localités JH, MG, OM, SS, KH, OH, KA. Th

\section{Atractylis cancellata L.}

Clairièrs des forêts, pâturages, champs incultes de la plaine et des basses montagnes. S, A, SA, SH, H. jusqu'au $1200 \mathrm{~m}$. localités SS, HB, OM, CL, OB, NW, AD, KH, KA, MG, KR. Th

\section{Bellis annua $\mathrm{L}$.}

Clairières des forêts, pâturages pierreux et sablonneux du littoral, des plaines et des basses montagnes. A, SA, SH, H. jusqu'au $1600 \mathrm{~m}$. localité OT. Th

\section{Bellis sylvestris Cyr.}

Forêts et pâturages de la plaine et des basses et moyennes montagnes calcaires et siliceuses. 
SA, SH, H. jusqu'au 2350 m. localité OT. Hém

\section{Calendula arvensis $\mathrm{L}$.}

Champs, lieux herbeux incultes. S, SA, SH. Localités OT, NW, AD. Th

\section{Carduus myriacanthus Salzm.}

Forêts claires, pâturages, champs sablonneux et rocailleux des plaines et des basses montagnes. A, SA, SH. Localités OM, OB, CL, OM, LM,AG, SS, OT, JH. Th. EMI.

\section{Carlina corymbosa L.}

Pâturages pierreux de la plaine et des montagnes, clairières des forêts. A, SA, SH. Jusqu'au 2000 m. localités MG, KH, KA, CL, AG. Hém

\section{Carlina racemosa $\mathrm{L}$.}

Sol argilo-limoneux, humide d'hiver, en plaine et dans les basses montagnes, exceptionnellement dans les sous bois. A, SA, SH. Localités KH, OH, KA. Hém

\section{Centaurea africana Lamk.}

Forêts claires des plaines et des basses montagnes siliceuses. SA, SH, H. localités BH, KH, LM, KA. Hém

\section{Centaurea pullata L.}

Clairières des forêts, pâturages des plaines et des montagnes. A, SA, SH, H. jusqu'au 2200 m. localités SS, JH, HB, NW, OB, BA, KH, KR, LM, AS, KA, OT, CL. Hém (Th)

\section{Chamaeleon gummifer (L) Cass.}

Forêts claires, broussailles, pâturages de la plaine et des basses montagnes. A, SA, SH, H. localités SS, LM. Hém (G)

\section{Chamaemelum mixtum (L) All.}

Forêts claires, champs et pâturages sablonneux et pierreux de la plaine et des basses montagne. A, SA, SH, H. localités NW, KA, AD, $\mathrm{SB}, \mathrm{BA}, \mathrm{KH}, \mathrm{OH}, \mathrm{SS}, \mathrm{JH}$. Th $(\mathbf{C h})$

\section{Cichorium intybus L.}

Pâturages, champs incultes des plaines, des basses et moyennes montagnes. SA, SH, H. localités SS, CL. Hém

\section{Cirsium syriacum ( L.) Gaertn}

Bords des chemins, champs, lieux incultes dans les plaines et les basses montagnes. A, SA, SH. Localité non disponible. Th

\section{Cladanthus arabicus (L) Cass.}

Champs, pâturages arides. A, SA, SH. Jusqu' au $2000 \mathrm{~m}$. toutes les localités sauf TA, OH et BH. Th

\section{Cynara humilis L.}

Pâturages des terrains calcaires, siliceux, argileux, sablonneux, de la plaine et des basses montagnes. A, SA, SH. Jusqu'au $1450 \mathrm{~m}$. localités SS, JH, MG, OT, TA, CL, OB, HB, NW, $\mathrm{AD}, \mathrm{KH}, \mathrm{KR}, \mathrm{OM}, \mathrm{KA}$. G

\section{Echinops spinosus L.}

Forêts, pâturages, steppe dans les plaines et les montagnes. S, A, SA, SH, H. jusqu'au 2700 m. localités SS, MG, TA, OB, CL, KH. Hém (G)

\section{Evax pygmea (L.) Brot.}

Pâturages, pelouses sèches, sablonneuses et rocailleuses. A, SA, SH. Jusqu'au $1400 \mathrm{~m}$. toutes les localités. Th

\section{Filago germanica L.}

Forêts claires, pâturages pierreux argileux et sablonneux des plaines et des montagnes. S, A, SA, SH, H. jusqu'au $1050 \mathrm{~m}$. toutes les localités. Th

\section{Galactites tomentosa Moench}

Pâturages, clairières des forêts, champs, lieux incultes, bords des chemins. SA, SH, H. localité non disponible. Th

Hedypnois rhagadioloides (L.) F.W. Schmidt

Forêts claires, pâturages et cultures dans les plaines et les montagnes. SH. Jusqu'au $1600 \mathrm{~m}$. localités OB, CL. Th

\section{Hypocheris glabra L.}

Forêts claires, pâturages sablonneux et pierreux dans les plaines, et les basses et moyennes montagnes calcaires et siliceuses. SA, SH. Localité non disponible. Th

\section{Leontodon hispidulus (Del.) Boiss.}


Pâturages rocailleux et sablonneux arides. A, SA, SH. Localités KH, MG. Th

Leontodon maroccanus (Pers) Ball.

Pâturages sablonneux et argileux des plaines et des basses montagnes. A, SA, SH. Jusqu'au $1200 \mathrm{~m}$. toutes les localités sauf TA et OH. Th. EMA.

\section{Leontodon saxatilis Lamk.}

Pâturages sablonneux, argileux et pierreux, clairières des forêts, marais des plaines et des basses et moyennes montagnes. A, SA, SH. Localités SS, NW, MB, JH, MB, OM, OB, CL, HB, OT, KA, LM, KR, BA, AD. Hém

\section{Leotodon taraxacoides (Vill) Merat.}

Forêts, clairières, pâturages sablonneux, argileux et pierreux, marais des plaines et des basses et moyennes montagnes. A, SA, SH, H. localités SS, NW,MB, JH, MG, OM, OB, CL, HB, OT, KA, LM, KR, BA, AD. Th (Hém)

\section{Leontodon tuberosis L.}

Clairières des forêts, pâturages des plaines et des basses et moyennes montagnes. A, SA, SH. Localité OM. Hém

Logfia gallica (L) Cosson \& Germ.

Forêts claires, champs et pâturage sablonneux ou pierreux des plaines et des montagnes siliceux. SA, SH, H. jusqu'au 2500 m. localités SS, MG, MB, OM, JH, SM, BH, MG, CL, OB, NW. Th

Mantisalca salmantica (L) Briq et Cavill.

Clairières des forêts, pâturages de la plaine et des montagnes. A, SA, SH. Jusqu'au $1850 \mathrm{~m}$. localités SM, OM, KH. Hém

\section{Pallenis spinosa (L) Cass.}

Forêts claires, pâturages des plaines et des montagnes. S, A, SA, SH, H. jusqu'au $2300 \mathrm{~m}$. localités OM, HB. Hém

\section{Phagnalon saxatile (L) Cass.}

Rochers, vieux murs, rocailles de la plaine et des montagnes. A, SA, SH, H. jusqu' au 2400 m. localités MG, SS, GH, MB, SM, OM, OB, HB, OM, CL, NW, BA, KH, KA, OT. Ch

\section{Scolymus hispanicus L.}

Clairières des forêts, pâturages, cultures des plaines et des montagnes sur tous terrains. S, A, SA, SH, H. jusqu'au $1600 \mathrm{~m}$. localités TA, SS, OB, CL, NW, AD, SB, OL, LM, KA, OT, AG. Hém

Senecio leucanthemifolius Poiret

Clairières des forêts, pâturages sablonneux et pierreux, sables et rochers maritimes, cultures des plaines et des montagnes. SA, SH, H. jusqu 'au 2500 m. localités SS, OT, KA, KH. Th'

\section{Sonchus oleraceus L.}

Très répandu dans les cultures, Plante très plastique, sans exigence édaphique. S, A, SA, SH, H. localités SS, TA, OM, AG, KH, OH, SB, KR. Hém

Tolpis barbata (L) Graetn.

Forêts claires, pâturages sablonneux et pierreux, dans les plaines, les basses et les moyennes montagnes. A, SA, SH, H. jusqu'au $2500 \mathrm{~m}$. localité non disponible. Th

\section{Boraginaceae}

\section{Anchusa italica Retz.}

Champs cultivés de la plaine et des montagnes. A, SA, SH. Jusqu'au $2300 \mathrm{~m}$. localités CL, HB, KH, AS, LM, OH, KA, AG. Hém

\section{Borrago officinalis L.}

Champs, pâturages argileux et rocailleux de la plaine et des basses montagnes. SA, SH, H. localité non disponible. Th

\section{Cynoglossum cheirifolium L.}

Clairières des forêts, pâturages rocailleux, plaine et surtout basses et moyennes montagnes. A, SA, SH, H. jusqu'au $2600 \mathrm{~m}$. localités JH, HB, SS, OT. Th

\section{Echium plantagineum L.}

Pâturages argileux et sablonneux, cultures, clairières des forêts. A, SA, SH, H. localités LM, SS, JH, MG, OT, SM, OM, OB, CL, HB, NW, AD, SB, KH, KR. Th 
Lithodora fruticosa (L.) Griseb.

Broussailles, clairières des forêts, rochers des collines littorales et des basses montagnes. SA. SH. Jusqu'au $1200 \mathrm{~m}$. localité non disponible. Ch. R.

\section{Lithospermum apulum (L.)}

Pâturages et champs incultes dans la plaine et les basses montagnes. SA, SH, H. jusqu'au 1700 m. localités HB, CL, BA, KH, KR, JH. Th

Nonnea vesicarea (L.) Reichenb.

Pâturages et cultures de la plaine et des basses montagnes. A, SA, SH. Localités OT, CL. Th

\section{Brassicaceae}

\section{Biscutella didyma $\mathrm{L}$.}

Pâturages, rocailles, bois, champs incultes, coteaux pierreux. A, SA, SH. Toutes les localités sauf BH et TA. Th

\section{Capsella bursa pastoris (L) Medic.}

Champs cultivés, lieux incultes dans la plaine et les montagnes. A, SA, SH, H. jusqu'au $3200 \mathrm{~m}$. localités SB, BA, OB, KH, OH, KA, LM. Th

Diplotaxis catholica (L.) DC.

Champs sablonneux, Broussailles, décombres, cultures, pâturages de la plaine et des basses montagnes. A, SA, SH, H. jusqu'au 1100 m. localités TA, OB, CL, SB, KH, MG, KA. Th

Eruca vesicaria (L.) Cav.

Champs, steppes, décombres. SA. Localités SS, OB, CL, NW, KH, KR, OM, LM, KA, SB. Th

\section{Raphanus raphanistrum susp. maritimus (Sm) Thell \\ Champs et jachères, décombres. A, SA, SH, H. localités JH, MG et TA. Th}

\section{Sinapis alba L.}

Champs, rocailles et rochers calcaires, vieux murs. A, SA, SH. Localité KH. Th

\section{Sinapis arvensis L.}

Champs, bords des chemins. A, SA, SH. Jusqu'au 1200 m. localités SS, OM, CL, OB, HB, LM, MG, OT. Th

\section{Campanulaceae}

\section{Campanula afra Cav.}

Forêts claires, pâturages pierreux, rocailles des plaines et des basses montagnes calcaires et siliceuses. SA, SH, H. jusqu' au $1500 \mathrm{~m}$. localités $\mathrm{OH}, \mathrm{AG}, \mathrm{LM}, \mathrm{KR}, \mathrm{KH}, \mathrm{SB}, \mathrm{BA}, \mathrm{AD}, \mathrm{NW}, \mathrm{OM}$, HB, OB, CL, MB, OT, SS. Th

Campanula fastigiata L. Duf.

Pâturages des terrains gypseux des hauts plateaux. SA, SH, H. localité KH. Th

\section{Campanula lusitanica $\mathrm{L}$.}

Forêts, broussailles, pâturages, rocailles de la plaine et des basses montagnes. SA, SH, H. jusqu'au $1200 \mathrm{~m}$. localité non disponible. Th. EMI.

\section{Campanula rapunculoides $\mathrm{L}$.}

Broussailles et forêts des plaines et des montagnes. SA, SH, H. jusqu' au 2000 m. localités JH, NW, KH, LM, KA. Hém

\section{Caprifoliaceae}

\section{Lonicera implexa Aiton.}

Forêts, broussailles, haies, plaines et montagnes. SA, SH, H. jusqu' au $2200 \mathrm{~m}$. localités AS, KH. Nph

\section{Caryophyllaceae}

\section{Corrigiola telephiifolia (Pourr.) Briq.}

Pâturages pierreux et sablonneux de la plaine, des basses et moyennes montagnes. SA, SH. Localité KH. Hém

\section{Herniaria hirsuta L.}

Champa sablonneux, lieux incultes arides. SA, SH. Jusqu' au 1200 m. localités NW, OB, SB, KH, KA. Hém. R.

\section{Paronychia argentea Lamk.}

Lieux incultes secs, pâturages de la plaine et des montagnes. A, SA, SH, H. jusqu'au 2300 
m. Toutes les localités sauf $\mathrm{OH}, \mathrm{AG}$ et BH. Hém

\section{Polycarpon tetraphyllum $\mathrm{L}$.}

Broussailles, forêts, cultures, terrains sablonneux ou pierreux, Alluvions des rivières, dans la plaine et les basses et moyennes montagnes. A, SA, SH, H. localité non disponible. Hém

Silene colorata Poiret

Clairières de forêts, pâturages, sables maritimes. SA, SH. Jusqu'au 2000 m. localité OH. Th

\section{Silene gallica L.}

Pâturages sablonneux et pierreux de la plaine, des basses et moyennes montagnes. SA, SH. Localités SS, NW, KH, KA. Th

Silene glauca (Spreng.) Pourret

Rochers, rocailles, broussailles de la plaine et des basses montagnes. SA, SH. Jusqu' au 1500 m. localité KA. Th

\section{Silene neglecta Ten.}

Broussailles de la plaine et des basses montagnes. SA, SH. Localités SS, CL, NW, AD, KH, LM, OH, KA. Th

\section{Silene vulgaris (Moench) Garcke}

Forêts claires, rocailles, moissons, champs incultes. A, SA, SH, H. jusqu'au 2200 m. localités LM, OH, KA. G

Spergularia marginata (DC.) Kittel.

Sables maritimes, terrains salés de l'intérieur. A, SA, SH, H. jusqu'au $1250 \mathrm{~m}$. localités SS, JH, MB, OM, SM, BH, OH, TA, MG, CL, KH, LM, AG, AS, KA. Hém

\section{Cesalpiniaceae}

\section{Ceratonia siliqua $\mathrm{L}$.}

Forêts de la plaine et des basses montagnes, ravins des montagnes. SA, SH. Jusqu'au $1600 \mathrm{~m}$. localité SB. Ph

\section{Cistaceae}

\section{Cistus albidus L.}

Broussailles des plaines et des basses montagneuses. SA, SH, H. jusqu' au $1100 \mathrm{~m}$. localités JH, SM, BH, TA, KH, LM, AG, OH, KA. Nph

\section{Cistus monspeliensis L.}

Forêts claires, broussailles, plaine et basses montagnes en terrains non calcaires. SA, SH. Jusqu'au 1300 m. localité M. Nph

\section{Cistus salviifolius L.}

Forêts claires, Broussailles, plaine et basses et moyennes montagnes en terrains non calcaires. SA, SH, H. jusqu'au $2100 \mathrm{~m}$. Toutes les localités sauf $\mathrm{OH}$ et $\mathrm{MB}$. Ch $\mathbf{( N p h )}$

\section{Cistus villosus $\mathrm{L}$.}

Forêts claires, pentes broussailleuses des basses et moyennes montagnes calcaires et siliceuses. SA, SH, H. jusqu'au 2100 m. localités OM, SS, JH, SM, HB, CL, AD, KH, KR, KA. Ch (Nph)

\section{Helianthemum ledifolium (L.) Miller}

Clairières des forêts, pâturages, champs incultes dans les plaines et les basses montagnes. S, A, SA, SH, H. jusqu'au 1150 m. localité non disponible. Th

\section{Helianthemum polyonthum (Desf.) Pers.}

Forêts claires, broussailles, pâturages des basses montagnes calcaires et siliceuses. SA, SH. Jusqu'au $1500 \mathrm{~m}$. localité non disponible.

Ch. EMA. R ?

\section{Tuberaria guttata (L.) Fourreau}

Forêts claires, pâturages sablonneux des plaines et basses et moyennes montagnes. Calcifuge. A, SA, SH. Localités OB, KR, CL, HB, $\mathrm{OM}, \mathrm{NW}, \mathrm{AD}, \mathrm{OB}, \mathrm{SS}, \mathrm{KA}, \mathrm{LM}, \mathrm{KH}, \mathrm{SB}, \mathrm{BA}$. Ch

\section{Clusiaceae}

\section{Hypericum perfoliatum $\mathrm{L}$.}

Forêts claires, broussailles de la plaine et des collines. A, SA, SH, H. localités HB, KH, LM, KA. Ch

\section{Hypericum perforatum $\mathrm{L}$.}

Forêts claires, broussailles de la plaine et 
des basses montagnes. S, A, SA, SH, H. jusqu'au 1800 m. localité KH. Ch

\section{Convolvulaceae}

\section{Convolvulus althaeoides L.}

Clairières des forêts, broussailles, champs incultes, rocailles, plaines et basses montagnes. S, A, SA, SH, H, HM. Jusqu'au 2900 m. localités SM, TA, LM. G

\section{Convolvulus arvensis $\mathrm{L}$.}

Cultures, pâturages, clairières des forêts, rocailles, plaines et basses et moyennes montagnes. S, A, SA, SH, H. jusqu'au $2400 \mathrm{~m}$. localités JH, SM, TA, OM, SS, OB, CL, AD, KH, KR, LM, AS, KA, HB. G

\section{Crassulaceae}

\section{Sedum dasyphyllum $\mathrm{L}$.}

Rochers calcaires et siliceux, murs, plaine et montagnes. SA, SH, H, HM. Jusqu'au 3000 m. localités JH, SS, MB, TA, CL, OM, NW, AD, OB, SB, BA, KH, AG, OH, KA. Ch

Umbilicus horizontalis (Guss.) DC.

Rochers, vieux murs, talus, sous-bois des plaines et des montagnes. A, SA, SH. Jusqu'au $1800 \mathrm{~m}$. Toutes les localités sauf JH, SM, BH, TA, HB et NW. G

\section{Cucurbitaceae}

Bryonia dioica Jacq.

Forêts et broussailles, haies, lieux frais ombragés de la plaine et des basses montagnes. A, SA, SH, H. localités JH et MG. G

\section{Cupressaceae}

\section{Tetraclinis articulata (Vahl) Masters.}

Forme des forêts et des broussailles dans la plaine et les basses montagnes. SA, SH. Jusqu'au 2000 m. localités MB, SS, OM, JH, OT, SM, BH, TA, MG, OM, OB, HB, KH. Ph

\section{Cuscutaceae}

Cuscuta epithymum (L.) L.
Parasite diverses plantes depuis la plaine jusqu'au hautes montagnes. S, A, SA, SH, H, HM. Jusqu'au 2000 m. localités CL, KH, OM, KA. Th

\section{Cyperaceae}

\section{Carex distachya Desf.}

Pâturages et broussailles de la plaine et des basses montagnes. SA, SH, H. jusqu' au $2000 \mathrm{~m}$. localités CL, KR, SB. Hém

\section{Dioscoreaceae}

\section{Tamus communis L.}

Forêts, ravins et broussailles. SA, SH, H. jusqu'au $1600 \mathrm{~m}$. localités SS, SM, MG, CL, SB, KH, AS. G

\section{Dipsacaceae}

\section{Lomeliosa stellata (L.) Rafin.}

Clairières des forêts, pâturages pierreux et argileux, plaines et montagnes. A, SA, SH, H. jusqu' au $1500 \mathrm{~m}$. localité non disponible. Th

\section{Scabiosa atropurpurea $\mathrm{L}$.}

Champs, pâturages sablonneux et rocailleux, broussailles et clairières de la plaine et des montagnes. A, SA, SH. Jusqu'au 2000 m. localité non disponible. $\mathbf{T h}$

\section{Ericaceae}

\section{Arbutus unedo L.}

Forêts et maquis de la plaine et des montagnes surtout siliceuses. SA, SH, H. jusqu'au 1900 m. localité KH. Nph (Ph)

\section{Euphorbiaceae}

\section{Euphorbia exigua L.}

Champs, pâturages sablonneux ou rocailleux de la plaine et des basses montagnes. SA, SH,H. localités OB, CL. Th

\section{Euphorbia falcata L.}

Champs, lieux incultes, pâturages sablonneux ou pierreux, plaine et basses montagnes. A, SA, SH, H. jusqu'au $1200 \mathrm{~m}$. localités JH, SS, TA, OB, CL, HB, OM, NW, SB, 
KH, KA. Th

\section{Euphorbia helioscopia L.}

Champs, bords des chemins, nitrophile. SH. jusqu' au $1600 \mathrm{~m}$. localités JH, AG, OT, SS, HB. Th

\section{Euphorbia medicaginea Boiss.}

Forêt, broussailles de la plaine et des montagnes. SA, SH, H. localité non disponible. Th

\section{Euphorbia pterococca Brot.}

Forêt, broussailles, lieux herbeux ombragés dans la plaine et les basses montagne. SA, SH. Localités JH, OT, CL, KA, HB, AS, LM, OH. Th

\section{Mercurialis ambigua $\mathrm{L}$.}

Lieux cultivés, bords des chemins, décembres, fissures des roches. $\mathrm{S}, \mathrm{A}, \mathrm{SA}, \mathrm{SH}, \mathrm{H}$. localités SS, MB, OM, JH, OT, SM, BH, TA, MG, NW, BA, SB, KH, AG, OH. Th

\section{Fabaceae}

Anthyllis hamosa Desf.

Forêts claires et pâturages sablonneux de la plaine. SA, SH, H. localité non disponible. Th

\section{Anthyllis tetraphylla $\mathrm{L}$.}

Pâturages des plaines et des montagnes, forêts claires. SA, SH, H. jusqu'au $2000 \mathrm{~m}$. localité KH. Th

\section{Anthyllis vulneraria L.}

Rochers des plaines et des montagnes calcaires et siliceuses, forêts claires, pâturages. SA, SH, H, HM. Jusqu'au 3000 m. Localités KH, OT, KA. Hém

\section{Astragalus glaux L.}

Forêts claires, pelouses sèches des collines et des basses montagnes. SA, SH. Localité non disponible. Hém

\section{Astragalus lusitanicus L.}

Forêts et Broussailles des terrains siliceux, plaines et basses montagnes. SA, SH. Localités HB, KH, AS, LM, AG. G

Coronilla scorpioides (L.) Koch.
Moissons, champs incultes, rocailles de la plaine et des basses montagnes. SA, SH, H. localités SM, HB, OB, AG, KH, OH, KA. Th

\section{Cytisus villosus Pourret}

Forêts fraîches, ravins humides du littoral et des montagnes siliceuses. SA, SH. Jusqu'au $1500 \mathrm{~m}$. localité non disponible. Nph

\section{Lathyrus angulatus L.}

Dayas, pâturages sablonneux humides de la plaine. SA, SH. Localité non disponible. Th

\section{Lathyrus cicera L.}

Champs, broussailles, pâturages de la plaine et des basses montagnes. A, SA, SH. Localités HB, KH. Th

\section{Lathyrus sphaericus Retz.}

Clairières des forêts, broussailles, pâturages de la plaine et des basses et moyennes montagnes. SA, SH. Jusqu'au 1800 m. localité KH. Th

\section{Lotus parviflorus Desf.}

Clairières et pâturages des terrains sablonneux. A, SA, SH. Localités OM, SM, CL, KH, LM, KA. Th

\section{Lupinus angustifolius $\mathrm{L}$.}

Champs, lieux sablonneux, de la plaine et des basses montagnes. A, SA, SH. Localité KH. Th

Lupinus pilosus Murr.

Forêts claires, pâturages sablonneux et rocailleux des terrains siliceux dans la plaine et les basses montagnes. A, SA, SH. Jusqu'au 1400 m. localités BH, OB, CL, KH. Th

\section{Medicago coronata (L.) Bartal.}

Clairières des forêts, pâturages, champs, bords des chemins, lieux herbeux, dans les plaines et les basses montagnes. SA, SH. Localités HB, AD. Th

\section{Medicago sativa L.}

Clairières des forêts, pâturages argileux et rocailleux des montagnes. SA, $\mathrm{SH}$. Localité non disponible. Hém

Ononis euphrasiifolia Desf. 
Terrains sablonneux. SA, SH. Localité non disponible. $\mathbf{T h}$

\section{Ononis mitissima $\mathrm{L}$.}

Forêts claires, pâturages un peu humides de la plaine et des basses montagnes. SA, SH. Localités MB, SS, JH, SM, HB, OM, KH, AS, LM, AG, OH. Th

\section{Ornithopus compressus L.}

Clairières des forêts, pâturages des terrains sablonneux de la plaine et des basses montagnes. A, SA, SH. Localités JH, SS, SM, CL, NW, BA, OB, KH, KR, LM, AS, OH, KA, SB. Ch

Sarothamnus arboreus (Desf.) DC.

Forêts et Broussailles, rocailles, plaine et montagnes. SA, SH, H. jusqu'au $1800 \mathrm{~m}$. localités CL, KH, AS, KA. Nph. R.

\section{Scorpiurus muricatus L.}

Champs et pâturages des plaines et des basses montagnes. A, SA, SH. jusqu'au $1050 \mathrm{~m}$. localité OT. Th

\section{Scorpiurus vermiculatus L.}

Champs, pâturages argileux et sablonneux de la plaine et plus rarement des basses montagnes. A, SA, SH, H. jusqu'au $1400 \mathrm{~m}$. localité TA. Th

\section{Trifolium angustifolium $\mathrm{L}$.}

Clairières des forêts, pâturages sablonneux et rocailleux de la plaine et des basses montagnes. A, SA, SH, H. jusqu'au $1700 \mathrm{~m}$. localités KR, $\mathrm{SB}, \mathrm{SS}, \mathrm{JH}, \mathrm{MB}, \mathrm{SM}, \mathrm{BH}, \mathrm{MG}, \mathrm{OM}, \mathrm{CL}, \mathrm{HB}$, $\mathrm{KH}, \mathrm{AS}, \mathrm{OH}, \mathrm{KA}$. Th

\section{Trifolium arvense $\mathrm{L}$.}

Clairières des forêts et pâturages surtout sablonneux dans la plaine et les basses montagnes. SA, SH, H. jusqu'au 2200 m. localité non disponible. $\mathbf{T h}$

\section{Trifolium campestre Schreber}

Champs sablonneux, lieux herbeux frais, plaine et montagnes. A, SA, SH, H. jusqu' au 2300 m. toutes les localités. Th
Clairières des forêts, pâturages de la plaine et des basses montagnes. SA, SH, H. localités SS, KA. Th

\section{Trifolium stellatum L.}

Clairières des forêts, broussailles, pâturages dans la plaine et les basses montagnes. SA, SH, H. jusqu'au $1800 \mathrm{~m}$. localités SS, JH, OT, CL, OM, NW, BA, KH, AS, KR, LM, AG, KA. Th'

\section{Vicia benghalensis L.}

Clairières des forêts, pâturages, champs incultes, moissons de la plaine et des basses montagnes. SA, SH. Localités NW, KH, KA. Th

Vicia disperma $\mathrm{DC}$.

Couteaux pierreux, broussailles, forêts de la plaine et des montagnes. SA, SH. Jusqu'au 1700 m. localités KH, OH, KA. Th

\section{Vicia lathyroides $\mathrm{L}$.}

Forêts et pâturages des montagnes, sols calcaires ou siliceux. SA, SH, H. jusqu'au 2500 m. localité KH. Th

\section{Vicia lutea $\mathrm{L}$.}

Pâturages, cultures de la plaine et des montagnes, clairières des forêts. SA, SH. Jusqu'au $2300 \mathrm{~m}$. localité non disponible. Th

\section{Vicia sativa L.}

Forêts claires, pâturages, broussailles, jachères des plaines et des montagnes. $\mathrm{SA}, \mathrm{SH}$, H. jusqu 'au 2400 m. localités CL, BA, KH, LM, AG, OH, KA.Th

\section{Fagaceae}

Quercus rotundifolia (Lam.) T. Morais.

Broussailles, forêts des moyennes montagnes. SA, SH, H. jusqu'au $2900 \mathrm{~m}$. Toutes leslocalités sauf SM, BH, TA. Ph

\section{Quercus suber L.}

Forêts surtout sablonneuses, plaines et montagnes bien arrosées. SA, SH, H. jusqu'au 1400. Localités SM, BA, KH, AS, LM, AG, OH, KA. Ph

\section{Gentianaceae}




\section{Centaurium erythraea Rafn.}

Forêts, Broussailles et pâturages, plaine et montagnes. A, SA, SH. Jusqu'au $2000 \mathrm{~m}$. localités NW, OM, SB, BA, OB, KH, KA, OH. Th (Hém)

\section{Geraniaceae}

Erodium laciniatum (Cav.) Wild.

Sables maritimes et sublittoraux, steppes, terrains arides rocailleux et sablonneux. A, SA, SH. Localités SM, SS, OT. Th

\section{Erodium malachoides ( L.) Wolld.}

Clairières des forêts, pâturages, champs des plaines et des basses et des moyennes montagnes. A, SA, SH. Localités KR, AG, KA. Th

Erodium moschatum (L) L'herit.

Pâturages, forêts claires, champs, chemins, lieux herbeux, dans la plaine et les basses montagnes. A, SA, SH. Localités SS, SM, CL, AD, BA, KH, AS, OH, KA, JH, SB. Th

\section{Erodium triangular (Forsk.) Musch.}

Steppes, terrains arides rocailleux Sables maritimes et sublittoraux. A, SA, SH.

\section{Localité KH. Th}

\section{Geranium molle L.}

Forêts claires, rocailles ombragés des montagnes. A, SA, SH, H. jusqu'au $1900 \mathrm{~m}$. localités SS, JH, OT, MG, MB, OB. Th

\section{Geranium robertianum $\mathrm{L}$.}

Forêt et rochers ombragés. A, SA, SH, H. localités SS, JH. Th

\section{Hyacinthaceae}

\section{Urginea maritima (L.)Baker}

Steppes, broussailles et pâturages pierreux ou sablonneux, rocailles et rochers gréseux ou calcaires de la plaine et des basses montagnes. A, SA, SH, H. jusqu'au $1400 \mathrm{~m}$. toutes les localités sauf TA. G

\section{Iridaceae}

Gladiolus segetum Kert-Gauwl.
Moissons, champs cultivés ou incultes de la plaine. A, SA, SH. Localités JH, KH, KA, SS. G

Iris sisyrinchuim L.

Lieux arides, pierreux ou sablonneux, de la plaine et des basses montagnes. SA, SH. Localités KA, SS, KH. G

Iris tingitana (Boiss \& Reuter.) B. \& T.

Pâturages et champs argileux un peu humides de la plaine. SA, SH. Jusqu'au $1700 \mathrm{~m}$. Localités KH, KA. G. EMA

\section{Juncaceae}

\section{Juncus conglomeratus L.}

Ruisselets et marais des plaines et montagnes siliceuses. A, SA, SH, H. localité CL. G

\section{Lamiaceae}

Ajuga iva (L) Schreb

Clairières des forêts, pâturages, champs incultes, rocailles calcaires et siliceuses, plaine et montagnes. A, SA, SH, H. jusqu'au $2700 \mathrm{~m}$. localités AS, HB, CL, KA, LM, KH, OB, NW, SM, OH. Hém (Th)

\section{Ballota hirsuta Bentham}

Forêts claires, rochers, broussailles, plaine et basses et moyennes montagnes. A, SA, SH, H. jusqu'àu $1200 \mathrm{~m}$. localité OB. Nph

\section{Lavandula multifida $\mathrm{L}$.}

Forêts claires, broussailles, pâturages arides, plaine et basses montagnes calcaires et siliceuses. A, SA, SH. Jusqu'au 1200 m. localités SS, OM, SM, BH, OB, CL, HB, SB, KH, KR, MG, JH. Ch

\section{Lavandula stoechas L.}

Forêts et broussailles des terrains siliceux, plaine et basses montagnes. SA, SH, H. localités $\mathrm{SS}, \mathrm{CL}, \mathrm{HB}, \mathrm{OM}, \mathrm{NW}, \mathrm{AD}, \mathrm{KH}, \mathrm{AS}, \mathrm{KR}, \mathrm{LM}$, OH, KA, OT. Ch

\section{Marrubium allysson L.}

Steppes, champs argileux. A, SA. Localité non disponible. $\mathbf{C h}$ 


\section{Marrubium vulgare $\mathrm{L}$.}

Clairières des forêts, pâturages, broussailles, cultures, plaine et basses et moyennes montagnes A, SA, SH, H. jusqu'au 1100 m. localités TA, SS, CL, KH. Ch

\section{Mentha aquatica $\mathrm{L}$.} Hém

Marais de la plaine. SA, SH, H. localité KH.

\section{Mentha pulgieum $\mathrm{L}$.}

Lieux humides de la plaine et des basses et moyennes montagnes. A, SA, SH, H. jusqu'au 2200 m. localités CL, KH, OT, SB. Hém (G)

\section{Mentha suaveolens Ehrt.}

Bords des eaux de la plaine et des montagnes. A, SA, SH, H. jusqu'au $2100 \mathrm{~m}$. localité KH. Hém

\section{Micromeria briquettinMaire}

Rochers calcaires des basses montagnes. SA, SA, SH. Localité SS, KH. Ch . EMA

\section{Nepeta tuberosa L.}

Clairières fraîches des forêts, prairies un peu humides des basses montagnes calcaires et siliceuses. A, SA, SH. Jusqu'au $2000 \mathrm{~m}$. localités CL, OB, MG, BH, TA, OM, OB, SS, HB. G

\section{Origanum compactum Benth.}

Forêts, broussailles et pâturages rocailleux de la plaine et des basses montagnes. A, SA, SH. Localités KH,TA. Ch. EMI.

\section{Phlomis crinita Cav.}

Clairières des forêts, pâturages pierreux des basses et moyennes montagnes calcaires et siliceuses. A, SA, SH. Localités SS, TA, HB, OB, $\mathrm{KH}, \mathrm{KR}, \mathrm{JH}$. Hém

\section{Prasium majus L.}

Forêts et broussailles, rochers, plaine et basses montagnes. A, SA, SH, H. jusqu'au 1300 m. localité non disponible. Nph

\section{Salvia verbenaca $\mathrm{L}$.}

Clairières des forêts, broussailles, pâturages, steppes, plaines et montagnes. A, SA, SH, H. jusqu'au 2500 m. Localités JH, KR, AS. Hém

\section{Stachys hirtus L.}

Champs, pâturages, terrains sablonneux de la plaine et des basses montagnes. SA, SH. Localités JH, OH, MB, SS, OT, SM, TA, OB, OM, NW, BA, KH, KR, LM, AG. Th

\section{Teucrium fruticans L.}

Forêts claire, broussailles, rochers, plaine et basses montagnes. SA, SH, H. jusqu'au 2150 m. localités SS, MB, JH, SM, TA, HB. Nph

\section{Ziziphora capitata L.}

Champs et pâturages pierreux secs. SA, SH, H. localités SS, KH. Th. RR.

\section{Liliaceae}

\section{Allium moly $\mathrm{L}$.}

Forêts et broussailles des basses montagnes siliceuses. A, SA, SH, H. localités MG, G. RR.

\section{Allium paniculatum $\mathrm{L}$.}

Broussailles, clairières des forêts, pâturages, champs incultes, rocailles calcaires et siliceuses, plaines et montagnes. A, SA, SH, H, HM. Jusqu'au 2700 m. localité KH. G

\section{Allium roseum $\mathrm{L}$.}

Broussailles, pâturages herbeux ou pierreux de la plaine et des basses montagnes. SA, SH, H. localités SS, SL, NW, AS, KH, SL. G

\section{Smilax aspera L.}

Haies, broussailles, rochers ombragés, maquis et forêts de la plaine et des basses montagnes. SA, SH, H. jusqu'au $1800 \mathrm{~m}$. localités MB, KH. Ph (G)

\section{Linaceae}

\section{Linum strictum L.}

Pâturages argileux et rocailleux de la plaine et des montagnes. SA, $\mathrm{SH}, \mathrm{H}$. localités $\mathrm{KH}, \mathrm{OH}$, KA. Th

\section{Linum usitatissimum L.}

Cultivé et subspontané ça et là, surtout dans l'ouest du pays. SA, SH, H. localités KH, KR, 
AG. Th

\section{Malvaceae}

Malva sylvestris L.

Champs et lieux incultes, décombres de la plaine et des montagnes. S, A, SA, SH, H, HM. Jusqu'au $2400 \mathrm{~m}$. toutes les localités sauf BH. Hém (Th)

\section{Oleaceae}

\section{Jasminum fruticans L.}

Forêts claires, broussailles, rochers, plaine, basses et moyennes montagnes. A, SA, H. jusqu'au $1600 \mathrm{~m}$. localités SM, TA, MG, MB, KH, SS, HB. Nph

Olea europaea subsp. oleaster (Hofmanns \& Link) Negodi.

Forêts et maquis des plaines et montagnes. A, SA, SH, H. jusqu'au $2000 \mathrm{~m}$. toutes les localités sauf TA et OT. Ph (Nph)

\section{Phillyrea latifolia L.}

Forêts et maquis, plaines et montagnes. SA, SH, H. jusqu'au $2200 \mathrm{~m}$. localités

MB, SS, OM, JH, SM, BH, TA, MG, OM, HB, KH, OT. Ph

\section{Orobanchaceae}

\section{Orobanche ramosa $\mathrm{L}$.}

Parasite de diverses plantes des plaines et des basses montagnes. A, SA, SH. Localités JH, OT, SM, OB. Th

\section{Papaveraceae}

\section{Papaver rhoeas $\mathrm{L}$.}

Champs, moissons, lieux incultes, dans la plaine, les basses et moyennes montagnes. A, SA, SH, H. localités SS, JH, TA, OB, CL, KH, HB. Th

\section{Plantaginaceae}

Plantago arenaria Waldst \& Kit.

Forêts claires, pâturages arides de la plaine et des basses montagnes. S, A, SA, SH, H. jusqu'au $1600 \mathrm{~m}$. localités JH, MB, SS, SM, TA, CL, OB, HB, NW, AD, SB, KH, KR, LM, KA, AG. Th. R?

\section{Plantago coronopus L.}

Clairières des forêts, dunes, pâturages terreux arides et humides et même salés des plaines jusqu'aux hautes montagnes. A, SA, SH. Jusqu'au 1500 m. localités MG, BH, GH, NW, AD, OB, OM, LM, OT. Th

\section{Plantago ovata Forsk.}

Steppes et pâturages désertiques, rocailles arides des basses montagnes. A, SA, SH. toutes les localités. Th

\section{Plumbaginaceae}

\section{Limonium lobatum (L. Fil) Chaz}

Pâturages arides, steppes des plaines et des montagnes. A, SA, SH. jusqu'au 1200 m. localité KH. Th

\section{Limonium sinuatum (L.) Miller}

Pâturages, forêts claires des terrains sablonneux du littoral, plaines et basses montagnes. S, A, SA, SH. Jusqu'au $3000 \mathrm{~m}$. localités SS, JH, OM, OT, SM, BH, TA, MG, OM, OB, HB, NW, AD, SB, KH, KA. Th

\section{Poaceae}

Aegilops ovata subsp triaristata (Wild) Roy

Champs et pâturages de la plaine et des basses montagnes. SH. jusqu'au $2200 \mathrm{~m}$. localités: OB, HB, BA, KH, OM, AS.'Th'

\section{Aira Caryophyllea L.}

Maquis, Broussailles, pâturages sablonneux de la plaine et des montagnes. SA, SH, H. jusqu'à 2200 m. localités NW, KA. Th

\section{Aira cupaniana Guss.}

Pâturages sablonneux, broussailles de la plaine et des montagnes. SA, SH, H. . jusqu'au 2100 m. localités KA, KH, OH. Th

\section{Anthoxanthum ovatum Lag.}

Pâturages, bords des dayas, broussailles et forêts de la plaine et des montagnes. SA, SH, H. 
jusqu'au 2800 m. localités KA, NW, AD, BA, KH, KR, LM, AG, OH. Th

Avena alba Vahl.

Clairières des forêts, cultures, pâturages sablonneux ou rocailleux de la plaine et des montagnes. A, SA, SH. Jusqu'au 2000 m. localités SS, KR, KH, KA. Th

\section{Avena sativa $\mathrm{L}$.}

Cultivé rarement. A, SA, SH. Localité KH. Th

\section{Avena sterilis $\mathrm{L}$.}

Moissons, bords des chemins, pâturages de la plaine et des basses montagnes. A, SA, SH. Jusqu'au 1500 m. localités SS, OM, CL, KH. Th

\section{Brachypodium distachyum (L) P.B.}

Rocailles et pâturages arides de la plaine et des montagnes. A, SA, SH, H. jusqu'au $2500 \mathrm{~m}$. localités SM, SS, OB, CL ,NW, SB. Th

\section{Briza maxima $\mathrm{L}$.}

Forêts des moyennes et hautes montagnes. SA, SH, H. jusqu'au 1400 m. localité KH. Th

\section{Briza minor L.}

Forêts des moyennes et hautes montagnes. SA, SH, H. localité KH. Th

\section{Bromus diandrus Roth.}

Forêts, pâturages, lieux sablonneux ou rocailleux de la plaine et des montagnes. A, SA, SH, H. localités SM, HB, OM, KH, KR. Th

\section{Bromus hordeaceusL.}

Forêts, champs, bords des dayas, pâturages de la plaine et des montagnes. A, SA, SH, H. jusqu'au 2400 m. localités JH, SS, OB, CL, KA. Th

\section{Bromus intermidius Guss.}

Pâturages et champs rocailleux, broussailles. A, SA, SH, H. localités T, R. Th

\section{Bromus lanceolatus Roth.}

Champs, moissons, pâturages de la plaine et des montagnes. A, SA, SH, H. jusqu'au 1900 $\mathrm{m}$. localité non disponible. Th

\section{Bromus madritensis L.}

Forêts et pâturages sablonneux et rocailleux de la plaine et des basses montagnes. A, SA, SH, H. jusqu'au $1800 \mathrm{~m}$. localité non disponible. Th

\section{Bromus rigidus Roth.}

Forêts, pâturages, lieux sablonneux ou rocailleux de la plaine et des basses montagnes. A, SA, SH, H. localité SS. Th

\section{Bromus rubens L.}

Steppes, moissons, pâturages sablonneux ou rocailles arides de la plaine et des basses montagnes. S, A, SA, SH, H. jusqu'au $1400 \mathrm{~m}$. localités MB, SS, CL, OB, NW, BA, KR, KH, AG. Th

\section{Bromus sterilis L.}

Forêts et pâturages. A, SA, SH, H. jusqu' au 2200 m. localités SS, MG, OM, CL, KH, HB. Th

\section{Cynodon dactylon $\mathrm{L}$.}

Champs, bords des chemins, décombres, lieux sablonneux de la plaine et des basses montagnes. S, A, SA, SH, H. jusqu' au $1200 \mathrm{~m}$. localités OL, OS, ES, R, T, CN, CS, WN, WS, SW, WD, ED, S, H, MA, GA, AA, AS. G

\section{Dactylis glomerata L.}

Pâturages, broussailles et forêts de la plaine et des montagnes. A, SA, SH, H. jusqu'au 2800 $\mathrm{m}$. localité non disponible. Hém

\section{Festuca caerulescens Desf}

Clairières des forêts. A, SA, SH, H. jusqu'au $1800 \mathrm{~m}$. toutes les localité sauf BH, SB, OT et JH. Hém

\section{Hordeum murinum L.}

Bords des chemins vieux murs, décembres, pâturages de la plaine et des montagnes. SA, SH. Jusqu'au 2000 m. localités CL, NW, SB, OB, LM, KA. Th

\section{Lamarckia aurea (L) Moenc}

Pâturages, champs, lieux incultes de la plaine et des montagnes. A, SA, SH. jusqu'au $2000 \mathrm{~m}$. toutes les localités sauf AS. Th

\section{Lolium rigidum Gaud}

Sables maritimes, champs, bords des dayas, 
rocailles de la plaine et des basses montagnes. A, SA, SH. Jusqu'au 1600 m. localités MG, CL, HB, LM, KA, GH. Th

\section{Lophochloa cristata (L.) Hyl.}

Steppes, champs incultes, broussailles, pâturages pierreux arides de la plaine et des basses montagnes. A, SA, SH. Jusqu'au $1900 \mathrm{~m}$. localités SS, MG, JH, OT, HB, AG. Th

\section{Phalaris minor L.}

Champs, moissons, pâturages sablonneux de la plaine. A, SA, SH. Localité non disponible. Th

\section{Poa annua L.}

Pâturages et lieux humides. SA, SH, H. jusqu'au $3400 \mathrm{~m}$. localité non disponible. Th

\section{Poa bulbosa L.}

Pâturages, lieux incultes, rocailles et rochers de la plaine et des montagnes. SA, SH, H. jusqu'au $2900 \mathrm{~m}$. localité non disponible. Th

\section{Polypogon monspeliensis (L.) Desf.}

Lieux sablonneux humides, de la plaine et des montagnes. A, SA, SH, H. jusqu'au $2000 \mathrm{~m}$. localités SS, OM, SM, OB, CL, HB, SB, KH. Th

Stipa capensis Thumb.

Steppes, champs incultes secs, pâturages pierreux arides de la plaine et des basses montagnes. A, SA. Jusqu'au $2000 \mathrm{~m}$. localités JH, SS, OM, SM, BH, TA, MG, OM, OB, CL, HB, NW, KR, KH, AD. Th

Vulpia alopecuros (Schousb.) (Link.)

Dunes, sables maritimes, champs et pâturages sablonneux de la plaine. A, SA, SH. Localités CL, AD, OB, AG, OH, KA. Th

Vulpia geniculata (L) Link Hort.

Pâturages et Broussailles. A, SA, SH. Localités non disponible. Th

\section{Vulpia myuros (L.) Gmel.}

Sables, pâturages secs, maquis de la plaine et des montagnes. A, SA, SH. 'Jusqu'au $1800 \mathrm{~m}$. localités SS, CL, OB, BA, AS, KH, OH, KA, AG. Th

\section{Polygonaceae}

Emex spinosa (L.) Campd.

Champs, lieux sablonneux, bords des chemins. S, A, SA, SH, H. localités TA, CL. Th

\section{Rumex bucephalophorus L.}

Terrains sablonneux ou pierreux de la plaine et des montagnes. A, SA, SH, H. jusqu'au 2100 m. Localités OT, SM, TA, CL, NW, BA, SB, OH, KA, JH, LM. Th

\section{Rumex pulcher L.}

Lieux humides, Dayas et Merjas. A, SA, SH. Localité CL. Hém

\section{Primulaceae}

\section{Anagallis arvensis L.}

Forêts claires, pâturages, Champs, rocailles, plaine, basses et moyennes et hautes montagnes. A, SA,SH, H, HM. Jusqu'au 2300 m. toutes les localités sauf MB. Th

\section{Rafflesiaceae}

Cytinus hypocistis (L.) L.

Parasite sur les Cistes. SA, SH, H. localités $\mathrm{SM}, \mathrm{AD}, \mathrm{OB}, \mathrm{KH}, \mathrm{AS}$; KR, LM, OH, KA. G

\section{Ranunculaceae}

\section{Clematis cirrhosa L.}

Maquis et forêts méditerranéennes surtout dans les régions bien arrosées (plus rares dans les régions sèches). Presque absent dans les régions steppiques et sahariennes. SA, SH, H. localités MB, SS, JH, OT, TA, HB. Ph

\section{Delphinium perigrinum L.}

Champs, pâturages, clairières des forêts des plaines et des montagnes. $\mathrm{SH}, \mathrm{H}$. jusqu'au 1600 $\mathrm{m}$. localité non disponible. Th

\section{Ranunculus aquatilis L.}

Eaux stagnantes de la plaine et des montagnes. SA, SH, H. jusqu' au 2700 m. localité KH. Th

Ranunculus bulbosus L. 
Clairières humides des forêts, marais, bords des ruiselets, dans les plaines et les montagnes. A, SA, SH, H,HM. Jusqu'au 2900 m. localités JH, SM, HB, AG, OT, CL, SB, KA, KH, KR. Hém

\section{Ranunculus paludosus Poiret.}

Clairières des forêts, pâturages des plaines et des montagnes. SA, SH, H. localité non disponible. Hém

\section{Resedaceae}

\section{Reseda lutea $\mathrm{L}$.}

Pâturages pierreux et sablonneux de la plaine et des basses montagnes. S, A, SA, SH, H. jusqu'au $2800 \mathrm{~m}$. localité CL. Th

\section{Reseda luteola L.}

Clairières des forêts, pâturages et champs, plaine et montagnes. SA, SH, H. jusqu'au 2400 m. localité non disponible. Th

\section{Rhamnaceae}

Ziziphus lotus (L) Lam.

Plaines argileuses, coteaux pierreux arides, steppes, dayas désertiques dans la plaine et les basses montagnes. S, A, SA, SH. Localité non disponible. Nph

\section{Rosaceae}

\section{Rosa sempervirens L.}

Forêts et broussailles fraîches de la plaine et des basses montagnes. A, SA, SH, H. jusqu'au 1200 m. localité KH. Ph

\section{Sanguisorba minor Scop.}

Forêts, broussailles et rochers de la plaine et des montagnes. SA, SH. jusqu'au $2600 \mathrm{~m}$. localités JH, CL, AD, OB, KR, OM, LM, KH, KA. Hém

\section{Rubiaceae}

\section{Galium verrecosum Hudson}

Rocailles, pentes pierreuses, moissons, lieux incultes secs des plaines et des basses et moyennes montagnes. SA, SH, H. localité KA. Th

\section{Galium viscosum Vahl.}

Clairières des forêts, pâturages, champs et jachères des plaines et des basses et moyennes montagnes calcaires et siliceux. A, SA, SH, H. jusqu'au $1300 \mathrm{~m}$. localités BH, TA, CL, KR, KA, SB, KH. Th

\section{Rubia peregrina $\mathrm{L}$.}

Forêts et broussailles de la plaine et des montagnes. A, SA, SH, H. jusqu'au $2000 \mathrm{~m}$. localités JH, OM, SS, SM, BH, NW, OB, KH, LM, KA. Ch (G)

\section{Sherardia arvensis L.}

Moissons, champs incultes, lieux herbeux de la plaine et des basses montagnes. A, SA, SH, H. localités SS, MG, GH, SM, CL, OB, HB, OM, NW, BA, KH, LM, AG, SB, KA, OH. Th

\section{Salicaceae}

\section{Salix alba L.}

Bords des eaux, lieux humides. A, SA, SH. Jusqu'au 2000 m. localité KH. Ph

\section{Santalaceae}

\section{Osyris lanceolata Hocht.}

Rochers calcaires et siliceux, broussailles de la plaine et des montagnes. SA, SH, H. jusqu'au 2000 m. localités OM, CL, KH, AS. Nph

\section{Scrofulariaceae}

\section{Anarrhinum fruticosum Desf.}

Rochers et rocailles des basses et moyennes montagnes calcaires et plus rarement siliceuses. SA, SH. Jusqu'au $2200 \mathrm{~m}$. localité non disponible. Ch . EMA.

\section{Anarrhinum laxiflorum Boiss.}

Forêts claires, pâturages pierreux, rocailles des moyennes montagnes calcaires et siliceuses. SA, SH. jusqu'au $2400 \mathrm{~m}$. localité non disponible. Hém. EMI. RR.

\section{Anarrhinum pedatum Desf.}

Rochers, broussailles et forêts, pâturage de la plaine et des basses montagnes. SA, SH. Jusqu'au $1500 \mathrm{~m}$. toutes les localités sauf SS, 
NW, BH et SB. Th. EMAT

\section{Linaria rharbensis Batt. Et Pitard}

Clairières des forêts, pâturages des champs des terrains sablonneux des plaines et des basses montagnes. SA, SH. Localité non disponible. Th. EMA

Misopates orontium (L) Rafin.

Forêts claires, pâturage, champs, rocailles, dans les plaines et les montagnes. A, SA, $\mathrm{SH}, \mathrm{H}$, HM. Jusqu' au 3000 m. localités CL, HB, OB, KH, KA, OT. Th

Parentucellia viscosa (L.) Caruel.

Bords des eaux, prairies marécageuses de la plaine et des montagnes. A, SA, SH. jusqu'au 2200 m. localités SS, JH, KH, KA. Th

\section{Scrofularia canina L.}

Rocailles, graviers des rivières dans les basses et moyennes montagnes, sables du littoral. A, SA, SH, H. localités TA, KH. Ch (Nph)

Veronica persica Poiret.

Culture, introduit et naturalisé. SA, SH. Endémique du Maroc. Localité non disponible. Th

\section{Solanaceae}

\section{Mandragora automnalis Spr.}

Broussailles, pâturages, champs, surtout dans les terrains argileux. A, SA, SH. Localité OT. G

\section{Thymelaeaceae}

\section{Daphne gnidium L.}

Broussailles, pâturages et forêts de la plaine et des montagnes. SA, SH, H. jusqu'au $2500 \mathrm{~m}$. localité non disponible. Nph

\section{Urticaceae}

\section{Parietaria mauritanica DR.}

Forêts, broussailles, haies, champs dans les terrains calcaires et siliceux, argileux et sablonneux. A, SA, SH. Localités SS, OM, CL, SB, KH, OT. Th

\section{Valerianaceae}

\section{Fedia cornucopiae (L.) Graetner}

Forêts claires, pâturages argileux, rocailleux et sablonneux des plaines et des basses montagnes.SA, SH, H. localités AG, OH, OT, MB, SS, BH, SM, TA, MG, CL, OB, HB, KH, AS, OM, LM. Th

\section{Valerianella carinata Lois.}

Pâturages rocailleux des montagnes. SA, SH, H. jusqu' au $2000 \mathrm{~m}$. Toutes les localités. Th

\section{Verbenaceae}

Vitex agnus-castus L.

Bords des ruisseaux et des rivières de la plaine et des basses montagnes. A, SA, SH, H. jusqu'au 1000 m. localités HB, OM. Nph

\section{Vitaceae}

\section{Vitis vinefera $\mathrm{L}$.}

Ravins humides et bordas des eaux. SA, SH, H. jusqu'au 1600 m. localité KH. Nph

\section{CONCLUSION}

L'analyse floristique de la forêt de l'Achach a permis de déceler la prédominance de certaines familles à savoir les Asteraceae, Poaceae, Fabaceae, Lamiaceae, Apiaceae, Caryophylaceae, Scrophulariaceae, Cistaceae, Brassicaceae et Boraginaceae. Ces familles comptent $63 \%$ de l'ensemble de la flore de la région de l'Achach.

Selon le catalogue des plantes vasculaires rares, menacées ou endémiques du Maroc, établi par Fennane et Ibn Tatou en 1998: - Trois espèces sont très rares, il s'agit de Allium moly, Anarrhinum laxiflorum et Ziziphora capitata. -Trois espèces sont rares, il s'agit de Herniaria hirsuta, Lithodora fruticosa, et Sarothamnus arboreus.-Deux espèces sont soupçonnées 
rares, à savoir Helianthemum polyontum et Plantago arenaria. Ces espèces rares sont d'un grand intérêt en matière de protection, d'estimation de la biodiversité et de la mise en valeur économique (Fennane et Ibn Tatou, 1998).

Sept espèces rencontrées dans la région de l'Achach sont endémiques du territoire Maroc-Algérie il s'agit de: Asparagus altissimus, Linaria rharbensis, Micromeria briquetti, Helianthemum polyontum, Anarrhinum fruticosum, Leontodon maroccanus et Iris tingitana. Six espèces sont connues comme étant endémiques pour le Maroc et la péninsule ibérique, il s'agit de Anarrhinum laxiflorum, Campanula lusitanica, Elaeosilinum foetidum, Origanum compactum, Asphodelus ramosus et Carduus myriacanthus. Deux espèces sont endémiques du territoire Maroc-AlgérieTunisie, c'est le cas de Anarrhinum pedatum et Aristolochia paucinervis.

L'aridité du milieu a favorisé un net développement des théropytes au dépens des autres types biologiques. Le spectre biologique est un spectre typique de l'ambiance bioclimatique semi-aride, avec un pourcentage de $52,78 \%$ pour les thérophytes, $10,4 \%$ pour les géophytes, $18,21 \%$ pour les hémicryptophytes, $7,43 \%$ pour les chaméphytes, $7,06 \%$ pour les nanophanérophytes et $4,08 \%$ pour les phanérophytes.

\section{BIBLIOGRAPHIE}

CHARAFI, Z. -1985- Cartographie au 1/ 100000 et caractérisation des sols dans la commune rurale d'OULED M'HAMED (Province de Settat). Mémoire de troisième cycle. Agronomie. IAV HASSAN II, Rabat, 134 p. COMBE, M., M. FERRE \& J.P. THAUVIN 1975- Méseta centrale et Méseta côtière. In Ressources en eau du Maroc, Tome 2.Ser. Géo. Maroc, Rabat.

FADLI, D. -1990- Evolution sédimentaire des massifs de M'dakra et du Khatouat, deux segments hercyniens de la Méseta marocaine Nord-occidentale. Doct. Es. Sc. Nat, Univ. Mohamed V, Rabat, 309 p.

FENNANE, M. \& M. IBN TATOU -1998Catalogue des plantes vasculaires rares, menacées ou endémiques du Maroc. Bocconea 8. Palerme. 243 p.

HANIFI, N., -1991- Importance des ressources phytogénétiques et leur utilisation en Algérie. In conservation des ressources végétales. Publication de Actes éditions. p47-49.

IBN TATOU, M. \& M. FENNANE -1991- Aperçu historique et état actuel des connaissances sur la flore vasculaire $d u$ Maroc. In conservation des ressources végétales. Publication de Actes éditions. p35-45.

MICHARD, A. -1976-Eléments de Géologie Marocaine. Serv. Géol., Rabat, n 300, 233 p.

NABLI, M.A. -1991- Diversité floristique en Tunisie. In conservation des ressources végétales. Publication de Actes éditions. $\mathrm{p}$ 51-52.

Aceptado para su publicación en julio de 2004

Dirección de los autores. Laboratoire de Botanique et de protection des Plantes. Faculté des Sciences. Kénitra. 